\title{
Medicina Baseada em Evidências: "novo paradigma assistencial e pedagógico"? \\ debates
}

\author{
Evidence Based Medicine: "a new paradigm for teaching and the provision of care?"
}

\author{
PALAVRAS-CHAVE: Medicina baseada em evidências; Educação médica; \\ condutas na prática dos médicos. \\ KEY WORDS: Evidence-based medicine; medical education; physician's \\ practice patterns. \\ PALABRAS CLAVE: Medicina basada en evidencia; educación médica; \\ conductas en la práctica de los médicos.
}

conductas en la practica de los médicos.

Luis David Castiel 1 Eduardo Conte Póvoa ${ }^{2}$

\section{Parte I}

A denominada 'medicina baseada em evidências' (MBE) originou-se do movimento da epidemiologia clínica anglo-saxônica, iniciado na Universidade McMaster no Canadá no início dos anos noventa. É definida em termos genéricos como o "processo de sistematicamente descobrir, avaliar e usar achados de investigações como base para decisões clínicas” (Evidence Based Medicine Working Group, 1992). Atualmente, a MBE está bastante em voga no âmbito biomédico, aí assumindo um papel de destaque, de tal modo que suas influências nas condutas médicas se manifestam significativamente. Basta consultar o Medline para obter profusas referências, atestando a difusão da proposta.

Vale ressaltar que os adeptos da MBE propõem uma escala tipológica da força das evidências que deve ser considerada para os processos decisórios nas práticas biomédicas.

\section{Tipos e Níveis de Evidências}

I- Evidência forte de, pelo menos, uma revisão sistemática (metanálise) de múltiplos estudos randomizados controlados bem delineados;

II- Evidência forte de, pelo menos, um estudo randomizado controlado bem delineado, de tamanho adequado e com contexto clínico apropriado;

III- Evidência de estudo sem randomização, com grupo único, com análise pré e pós-coorte, séries temporais ou casocontrole pareados;

IV- Evidência de estudos bem delineados não-experimentais, realizados em mais de um centro de pesquisa; V- Opiniões de autoridades respeitadas, baseadas em evidência clínica, estudos descritivos e relatórios de comitês de expertos ou consensos (Drummond \& Silva, 1998)

Temos, deste modo, a pretendida meta de aperfeiçoar o uso do raciocínio para além da casuística clínica de cada médico e de seus potenciais vieses. Para tal finalidade ser atingida, devem-se seguir determinados princípios, bem sintetizados por Jenicek (1997, p.189):

formulação de uma clara questão clínica a partir do problema do paciente que precisa ser respondido; busca na literatura por artigos relevantes e por outras fontes de informação; avaliação crítica da evidência (informação trazida por pesquisa original ou por síntese de pesquisas, p. ex. meta-análise); seleção da melhor evidência (...) para a decisão clínica; vinculação da evidência com experiência clínica, conhecimento e prática; implementação dos achados úteis na prática clínica; avaliação da implementação e do desempenho geral do profissional da MBE; ensino a outros médicos como praticar a MBE.

${ }_{1}^{1}$ Pesquisador do Departamento de Epidemiologia da Escola Nacional de Saúde Pública - FIOCRUZ. <castiel@ensp.fiocruz.br>

2 Coordenador do Curso de Pos-Graduação em Psicologia Médica e Psicossomática da Universidade Estácio de Sá, Rio de Janeiro. <econtepovoa@ig.com.br> 
Podemos resumir que a MBE se dirige a apoiar a experiência clínica com dados provenientes da epidemiologia clínica, complementadas com revisões sistemáticas da literatura, para critérios decisórios em condutas assistenciais. É recomendável o uso da informática médica como meio mais rápido e seguro de acesso ao que há de mais recente em termos de publicações. Na verdade, estas publicações são norteadas pelos centros ligados ao movimento da MBE, tais como o Cochrane Collaboration na Inglaterra. Os mesmos possuem uma rede de vínculos em várias partes do mundo, incluindo principalmente faculdades de Medicina.

No entanto, mesmo assumindo que as revisões do Cochrane são, em média, mais sistemáticas e menos enviesadas que estudos do mesmo teor publicados em outros periódicos, há investigações indicando que os resultados produzidos pelo Cochrane Collaboration também estão sujeitos a erros (incluindo problemas em métodos das análises). Esta foi a conclusão de uma meritória investigação realizada por participantes da seção nórdica do próprio grupo em relação a uma amostra de revisões de 1998. Muitas vezes, as conclusões dos trabalhos superestimavam benefícios de novas intervenções (Olsen et al., 2001).

\section{Parte II}

É preciso salientar a curiosa metamorfose gramatical ocorrida no processo de evolução da epidemiologia clínica para MBE. O adjetivo - 'clínica' se transforma em substantivo de maior amplitude 'medicina', e recebe uma locução adjetiva de indiscutivel efeito retórico. O 'baseada' veicula a sugestiva idéia de solidez, como 'fundação/fundamento' que será constituído pelo elemento sensorial humano considerado mais fidedigno (a visão) para testemunhar-se fatos que poderão ser, então, evidenciados como 'verdades' (Castiel, 1999)

Independente dos aspectos discursivos, a MBE tem sido alvo de intenso debate nos domínios das ciências da saúde (Jenicek, 1997; Jackson et al, 1998). Não é nossa intenção detalhar aqui as múltiplas discussões acerca do campo em foco com críticas de caráter ético, conceitual e metodológico.

Porém, vale destacar a problemática integração com a experiência clínica, conhecimento e prática de cada médico. Principalmente, porque não são explicitadas no movimento MBE que as formas de operar categorias na biomedicina podem se dar mediante modos de raciocínio que podem eventualmente se superpor - hipotético-dedutivo, indutivo e abdutivo - em diferentes momentos do processo clínico.

Tomemos o fato de textos produzidos pelo movimento da MBE anunciarem tratar-se de um "novo paradigma assistencial e pedagógico" (grifos nossos) (Sackett et al., 1997). Alguns explicitam tal propriedade como um subtítulo na própria capa do livro (Drummond \& Silva, 1998). Como mostram Sayd e Nunes-Moreira (2000), em um pertinente texto sobre as possíveis afinidades propositivas encontradas no pensamento filosófico cético e no movimento da MBE, esta não constituiria novo paradigma, pois, consiste em um modelo cognitivo adaptado à prática clínica que não rompe com modos lógico-racionais de produção de conhecimento científico.

Importa, agora, salientar que tal noção kuhniana sofreu uma impressionante popularização, tornandose, de certa forma, um termo abusivamente empregado, não obstante sua polissemia. Mesmo no campo da filosofia da ciência sua imprecisão semântica já foi discutida e, inclusive, reelaborada pelo próprio Kuhn (1974).

Cabem aqui alguns comentários sobre o uso desgastado da idéia de 'paradigma' não apenas nos domínios acadêmicos mas, também, para além de suas fronteiras. Jesus de Paula-Assis (1993) mostra como a importação de termos kuhnianos - especialmente da 'Estrutura das revoluções científicas' (Kuhn, 1970) - por parte de autores das ciências sociais e humanas é pouco fiel às intenções de Kuhn. Acreditamos que isto vale também para o uso feito pelos autores e divulgadores da MBE. Kuhn é um teórico e crítico da racionalidade científica e propõe um modelo de análise para a racionalidade humana, seus efeitos e consequëncias com base nas ditas ciências naturais. Estas, por sua vez, constituem, cada vez mais, elementos centrais da cultura ocidental com vistas à produção de conhecimento e de objetos técnicos.

Como já foi indicado (Castiel, 1999), o enfoque da MBE permanece eminentemente vinculado às tradições da razão cientificista. Aliás, não poderia ser de outra forma, pois a prática médica se vê como uma disciplina diretamente vinculada às biociências. Apenas, porém, há uma alteração de enfoque, com a ênfase nos modos empiricistas de investigação na ciência, veiculados especialmente pelos estudos da epidemiologia clínica ao abordar dimensões diagnósticas/terapêuticas/prognósticas na prática biomédica. Em outras palavras, se a Medicina é vista por alguns como uma mescla de 'ciência' e 'arte' (poder-se-ia até dizer 'artesanato', no qual o mestre-artesão se constituiria como autoridade, expert (perito) na sua especialidade), a MBE reforçaria bem mais a dimensão 'ciência', para evitar os riscos de erros em 
decisões baseadas em intuições originárias na experiência acumulada, conferida somente pela mestria nas 'artes clínicas'.

\title{
Parte III
}

Um importante aspecto nesta discussão diz respeito à autonomia do paciente que pode ser levada em conta, mas, no entanto, fica subjacente à primazia de abordagens objetivantes, universalizantes e previamente qualificadas - a escolha do médico junto a seu paciente passaria a ser a escolha da "melhor evidência", prevalecendo, em tese, o princípio bioético da beneficência.

Podemos utilizar o conceito de "função apostólica" que Balint (1998) atribuiu aos médicos quando pretendem impor regras a seus pacientes. A diferença parece ser que, a partir dos parâmetros para se atingir a "melhor evidência", o médico também é influenciado por outros parâmetros: aqueles avalizados pela expertise em MBE. Ou seja, ele próprio deve alcançar níveis de expertise - baseada em evidências mediante recursos de pesquisa via informática médica.

Parece que a consideração da subjetividade do paciente perde cada vez mais seu espaço. Parâmetros, protocolos, meta-análises, dados epidemiológicos, são de extrema relevância na produção de conhecimento e, portanto, de teoria médica, mas não deverão substituir a arte do cuidar que demanda afinidades empáticas, habilidades intuitivas, para, eventualmente, em determinado caso, abrir mão da incorporação de "evidências".

O que vale questionar não é evidentemente a gama de ferramentas que o avanço tecnológico traz para o arsenal diagnóstico e terapêutico, mas se toda esta corrida em direção a evidências científicas baseadas em dados estatísticos, epidemiológicos e a valorização da informática médica, não seria a tentativa de transformar metonimicamente partes da Biomedicina em toda esta.

Podemos perceber que grande parte das propostas feitas pelos defensores da MBE, constitui evolução de um mesmo tipo ideal, ou seja, de uma mesma racionalidade médica organizada em torno do caráter cientificista, com vistas a parametrizar a prática médica, mantendo a doença como entidade nosográfica tal como é categorizada pelos manuais taxonômicos como objeto de diagnóstico e intervenção em detrimento da valorização da singularidade da saúde humana em sua hipercomplexidade (Morin, 1990).

Pelo menos no que tange ao conceito kuhniano de paradigma, a MBE está longe de ser a proposta de um novo paradigma. Se esta proposta critica com pertinência a forma infundada de (muitas vezes) que determinados médicos se utilizavam para decidir por determinadas práticas, da mesma forma, pode, muitas vezes, cercear médicos no exercício de sua capacidade clínica intuitiva, empática, para perceber aquilo que não é da ordem do concreto, do objetivamente evidenciável.

Com estas considerações não se pretende propor a abstenção de métodos científicos que possam ajudar na produção de conhecimentos clínicos. O que se procura enfatizar é o modelo hegemônico em que predomina a pretensa ilusão de que a Medicina Ocidental seria levada a 'verdades', orientada por uma "soberania" científica. É perceptível a "nuvem de fumaça" que o objetivismo e o modelo mecanicista da Biomedicina vem deixando diante de aspectos subjetivos, psicológicos e sócio-culturais dos pacientes, permanecendo como objeto principal a preocupação com a DOENÇA.

Camargo-Jr. (1992, p.10-1) comenta:

\begin{abstract}
Penso que supor, como Kuhn, a ciência como um empreendimento apenas parcialmente como determinante fundamental na forma como o cientista percebe o mundo, abre perspectivas no estudo do que chamei de paradoxos da clínica. Refiro-me em especial ao papel condicionante que as teorias correntes acerca das categorias diagnósticas e de sua gênese têm no modo como o médico traduz o sofrimento que seus pacientes apresentam, supervalorizando os aspectos objetiváveis, traduzidos em doença, e deixando de lado o universo subjetivo do sofrer.
\end{abstract}

Em outras palavras, nossa argumentação propõe que as proposições da MBE não trazem mudanças de paradigma à Medicina. Poderíamos até ousar dizer que se trata de uma tentativa de ratificar a racionalidade médica ocidental já existente, por meio da sofisticação e refinamentos de objetos e técnicas, segundo os cânones dos empreendimentos científicos.

\section{Parte IV}

Como diz Rushton (2001, p.349) em uma pertinente crítica à MBE:

Muitas vezes a resposta baseada em evidências a maioria destas questões não é clara ou a 'evidência é incompleta'. De alguma forma, em nossa volúpia dos dados duros (hard data), estas 


\title{
DEBATES
}

\author{
respostas desconhecidas transformam-se em 'não'. Se não há clara e convincente evidência, o \\ modo 'automático' (default) é encarar a prática como sem valor. Reduções drásticas em despesas \\ hospitalares e recusa de pagamentos utilizam esta abordagem para cortar duramente atividades \\ destituídas do impossível, muitas vezes inalcançável, peso da evidência.
}

É preciso reiterar o hiperdimensionamento que se tenta dar a uma faceta da biomedicina (entre várias), tanto no que se refere a sua prática e ao ensino, assim como a seu desdobramento no campo semântico (o método se transforma em toda uma Medicina). Há, portanto, que se ter muito cuidado quando se propõe a mudança de um paradigma pedagógico para a formação médica: em primeiro lugar, pelos aspectos já referidos, em segundo lugar, pelo que se pretende dizer epistemologicamente com a palavra "paradigma", e, em terceiro lugar, pela necessidade de se colocar as seguintes questões: Do que é que a Biomedicina mais carece? Ou, ainda, de que tipo de treinamento nossos médicos precisam para estar atentos tanto à qualidade de vida pessoal quanto às intervenções técnicas (não sendo possível descurar de aspectos subjetivos de seus pacientes e suas famílias)? Qual seria a dimensão da saúde a ser alcançada?

Se é verdade que nossa formação médica é ainda predominantemente cartesiana e que sua racionalidade científica vem reduzindo a percepção da importância da complexidade humana, como incluir elementos para lidar com as limitações que outras disciplinas/saberes sinalizam (como a Antropologia Médica, a Psicanálise) para abordar tais aspectos, diante de mais esforços dirigidos para um conhecimento baseado em supostas verdades científicas?

Certamente estamos diante de um desafio muito maior: pensar em saúde de forma complexa - isto é, levando em consideração suas múltiplas dimensões: subjetivas, sócio-culturais, biológicas, sem desconsiderar evidentemente a vertente científica e o que ela tem a nos oferecer. Mas vale a pena ressaltar que diante da perplexidade e das incertezas humanas, o método científico mais acurado acaba por ser apenas um dos modos de abordar e ordenar o real. Cabe aos profissionais da área de saúde cogitarem que a ampliação conseqüente do cuidado em saúde talvez só seja possível por meio de significativas superações - de vaidades, de preconceitos, do corporativismo, da falta de compaixão pelo sofrimento humano. Em suma, há necessidade de uma ética compartilhada entre profissionais de saúde e sociedade civil.

Além disto, possivelmente diante da força retórica da expressão, presencia-se a proliferação de atividades propostas baseadas em evidências (BE). Mesmo assumindo que a epidemiologia sempre atuou como fonte de evidências para a saúde pública, ainda assim, propugnam-se uma 'saúde pública BE' (Muir Gray, 1997), 'processos decisórios para programas de saúde comunitária BE' (Jackson et al., 1998), promoção à saúde BE, (Jenicek, 1997) e, também, uma 'escolha por parte dos pacientes BE' (Eysenbach \& Diepgen, 2001).

\section{Parte V}

Apesar das contribuições das atividades técnicas na Medicina, que procura basear-se em evidências, parece existir uma sobrecarga de busca de evidências na relação médico-paciente, em detrimento de tal relação. Isto pode ser ilustrado em um significativo estudo qualitativo empregando grupos 'estilo Balint' (sem a presença de psicanalista) com 'general practitioners' ingleses (Freeman \& Sweeney, 2001). Os achados mostraram que há circunstâncias que interferem na implementação de ações médicas BE. Por exemplo: as experiências pessoais e profissionais do médico; a relação que o médico estabeleceu com seu paciente individual e a percepção da evidência em relação ao caso específico; a forma de se expressar nas consultas pode induzir pacientes a aceitar ou rejeitar evidências clínicas; as condições logísticas de internamento, do tratamento, da gravidade do caso, das situações pessoais dos pacientes podem influenciar na adoção de medidas BE. Além disto, há uma tensão entre médicos de atenção primária e da atenção secundária em lidarem com as abordagens BE. Há uma percepção que especialistas seguiriam mais facilmente protocolos $\mathrm{BE}$.

A nosso ver, os obstáculos maiores não se referem às dificuldades provenientes de, nem sempre, dispor-se de evidências seguras sobre determinadas questões, ou, mesmo quando estão disponíveis, não haver certeza sobre quais seriam as melhores escolhas. Ou, ainda, problemas em se operar com variáveis (in)determinadas ou nas limitações na implementação de medidas de promoção à saúde. Preocupações centrais deveriam, na medida do possível, tomar como base a questão de a saúde se configurar como uma macrocategoria multifária. Pois admite, conforme os contextos, múltiplas definições, com distintas repercussões e decorrências. Especialmente, no âmbito humano, apesar das dificuldades de defini-la, 'saúde', certamente, é distinto de 'doença', mas também é diferente de 'vida'. A saúde seria um pré-requisito para o 'viver', que, inclui o prazer, a dor, a invenção, a criatividade, os arrebatamentos. $\mathrm{E}$ 'viver', infelizmente, não é passível de ser baseado em evidências. 


\section{Referências}

BALINT, M. O médico, seu paciente e a doença. Rio de Janeiro: Atheneu, 1988.

CAMARGO-JR, K. R. Paradigmas, ciência e saber médico. Rio de Janeiro: Cadernos do Instituto de Medicina Social, 1992.

CASTIEL, L. D. A medida do possível... Saúde, risco e tecnobiociências. Rio de Janeiro: Ed. Contracapa/Ed. Fiocruz, 1999.

DRUMMOND, J. P., SILVA, E. Medicina baseada em evidências. Novo paradigma assistencial e pedagógico. Rio de Janeiro: Atheneu, 1998.

EVIDENCE-BASED WORKING GROUP. Evidence-based medicine. A new approach to teaching the practice of medicine. JAMA, v.268, p.2420-5, 1992.

EYSENBACH, G., DIEPGEN, T. L. The role of e-health and consumer health informatics for evidence-based patient choice in the 21st century. Clin. Dermatol., v.19, p.11-7, 2001.

FREEMAN A. C., SWEENEY, K. Why general practitioners do not implement evidence: qualitative study. BMJ, p.323, p.1-5, 2001.

JACKSON, C. A., PITKIN, K., KINGTON, R. Evidence-based decisionmaking for community health programs. Washington: Rand, 1998.

JENICEK, M. Epidemiology, evidenced-based medicine, and evidence-based public health. J. Epidemiol., v.7, n.4, p.187-97, 1997.

KUHN, T. A estrutura das revoluções científicas. São Paulo: Perspectiva, 1970.

KUHN, T. Second thoughts on paradigms. In: SUPPE, F. (Org.) The structure of scientific theories. Chicago: University of Illinois Press, 1974. p.7-28.

MORIN, E. Ciência com consciência. Lisboa: Publicações Europa-América: Biblioteca Universitária, 1990.

MUIR GRAY, J.A. Evidence-based public health - what level of competence is required? J. Public Health Medicine, v.19, n.1, p.65-8, 1997.

OLSEN, O., MIDDLETON, P., EZZO, J., GOTZSCHE, P.C., HADHAZY, V., HERXHEIMER, A.,

KLEIJNEN, J., MCINTOSH, H. Quality of Cochrane reviews: assessment of sample from 1998. BMJ, v.323, p.829-32, 2001.

PAULA-ASSIS, J. Kuhn e as ciências sociais. Estudos Avançados, v.7, n.19, p.133-64, 1993.

RUSHTON, J. L. The burden of evidence. BMJ, v.323, p.349, 2001.

SACKETT, D. L., RICHARDSON, W.S., ROSENBERG, W., HAYNES, R.B. Evidence-based medicine. How to practice and teach EBM. London: Churchill Livingstone, 1997.

SAYD, J. D., NUNES-MOREIRA, M. C. Medicina baseada em evidências - Ceticismo terapêutico, recorrência e história. Physis, v.10, p.1, 2000.

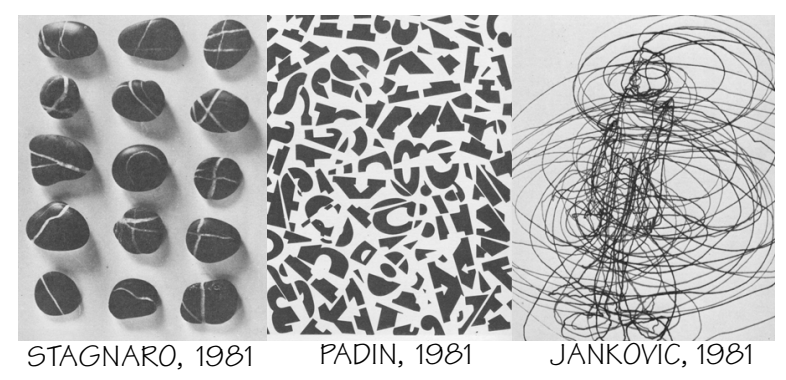

Recebido para publicação em: 16/01/02 Aprovado para publicação em: 22/02/02 